\title{
UNIQUE SUBDIRECT SUMS OF PRIME RINGS(1)
}

\author{
BY \\ LAWRENCE LEVY
}

1. Introduction. The purpose of this paper is to introduce a type of subdirect sum which possesses strong uniqueness properties. Intuitively speaking, a subdirect sum is irredundant if none of the summands can be omitted (without producing a proper homomorphism). This definition is made more precise in $\$ 2$.

In $\$ 3$, the first main result characterizes all irredundant subdirect sums of prime rings as those semi-prime rings $R$ in which every annihilator (2-sided) ideal $(\neq R)$ is contained in a maximal annihilator ideal. It is shown that the kernels of the projections $R \rightarrow R_{\alpha}$ are uniquely determined ideals of $R$. This not only implies uniqueness, up to isomorphism, of the components $R_{\alpha}$, but also of the structure of the subdirect sum combining them. We next consider the question of decomposing an $R$-module $M$ ( $R$ as above) into a subdirect sum of modules $M_{\alpha}$ over the component rings $R_{\alpha}$. We show that this is not always possible, and then we identify a class of modules for which the decomposition exists and is unique. By-products of the investigation are the result that the lattice of annihilator ideals of $R$ is isomorphic to the lattice of subsets of the index set $\{\alpha\}$, and a characterization of all subdirect sums of a finite number of prime rings.

In $\$ 4$ we show that the injective envelope of $M(M, R$ as above) is completely determined by the components $\left\{M_{\alpha}\right\}$ and $\left\{R_{\alpha}\right\}$ and is therefore independent of the two subdirect sunıs involved. We also show that the maximal ring of right quotients of $R$ is the (complete) direct product of the maximal rings of right quotients of the component rings $R_{\alpha}$.

In $\$ 5$ we consider analogues, for subdirect sums, of the so-called "square root" (if $A \oplus A \cong B \oplus B$, is $A \cong B$ ?) and "cancellation"' (if $A \oplus C \cong B \oplus C$, is $A \cong B$ ?) problems.

In the last section we consider the preservation of the existence of a classical quotient ring under subdirect sums and decompositions, and we prove that a ring has a right quotient ring which is semi-simple with minimum condition if

Presented to the Society November 25, 1960 under the title Rings with semi-simple quotient rings. Preliminary Report; received by the editors April 29, 1961 and, in revised form, December 12, 1961.

(1) This paper grew out of a doctoral dissertation submitted to the University of Illinois. The author would like to express his appreciation for the assistance given to him by Professor I. Reiner. 
and only if it is an irredundant subdirect sum of a finite number of rings which have simple (with minimum condition) right quotient rings, the kernels of the projections again being unique.

2. Irredundant subdirect sums. Let a set of rings $\left\{R_{\alpha}: \alpha \in A\right\}$ be given. A ring $R$ is a subdirect sum of $\left\{R_{\alpha}\right\}$ if there is an isomorphism $h$ of $R$ into the (complete) direct product $\prod_{\alpha} R_{\alpha}$ such that each of the induced projections $r \rightarrow h(r)_{\alpha}$ maps $R$ onto $R_{\alpha}$. The subdirect sum is irredundant if for each $\beta \in A$, the kernel of the map $h_{(\beta)}: r \rightarrow\left\{h(r)_{\alpha}: \alpha \neq \beta\right\}$ of $R$ into $\prod_{\alpha \neq \beta} R_{\alpha}$ is nonzero. We adopt the convention that the direct product of the empty set of rings is 0 .

The definition of irredundance implies that $R_{\alpha} \neq 0$ for each $\alpha \in A$, that each nonzero ring is an irredundant subdirect sum of itself, and that 0 is an irredundant subdirect sum of the empty set of rings.

If we identify $R$ and $h(R)$ (which we will do without explicit mention), then a subdirect sum $R$ of the rings $\left\{R_{\alpha}\right\}$ is irredundant if and only if $R \cap R_{\beta} \neq 0$ for each $\beta$ : for $R \cap R_{\beta}=\operatorname{ker} h_{(\beta)}$.

Finally, we observe that $R$ is an irredundant subdirect sum of the set of rings $\left\{R_{\alpha}\right\}$ if and only if there exists a set of ideals $\left\{P_{\alpha}\right\}$ of $R$ such that $R_{\alpha} \cong R / P_{\alpha}$, $\bigcap_{\alpha} P_{\alpha}=0$, and for each $\beta, \bigcap_{\alpha \neq \beta} P_{\alpha} \neq 0$.

An example of an irredundant subdirect sum is $R=\{(a, b): a, b \in Z, a-b \in 2 Z\}$ where $Z$ is the ring of integers. Then $R \cap(Z, 0)=(2 Z, 0)$ and $R \cap(0, Z)=(0,2 Z)$, so that the sum is irredundant but not direct.

An obvious modification of the above discussion holds for subdirect sums of modules over a given ring.

3. Main decomposition theorems. A ring is prime if $A B=0$ for (2-sided) ideals $A, B$ implies that $A$ or $B=0$. A ring is semi-prime if it has no nonzero nilpotent ideals.

Let $R$ be a ring and $M_{R}$ a right $R$-module. For each (2-sided) ideal $J$ in $R$, set $\operatorname{ann}_{M} J=\{m \in M: m J=0\}$, and call every submodule of the form $\operatorname{ann}_{M} J$ an annihilator submodule. Similarly, define $\operatorname{ann}_{R} N$ for $N_{R} \subseteq M$. The annihilator submodules of $R_{R}$ will be (2-sided) ideals and we will call these the annihilator ideals of $R$. A maximal (resp. minimal) annihilator submodule of $M$ is a maximal (resp. minimal) element of the set of annihilator submodules of $M$ different from $M$ (resp. different from 0 ).

If $J$ is an ideal in a semi-prime ring $R$, then the left and right annihilators of $J$, in $R$, have zero intersection with $J$ (the squares of these intersections are zero), and hence they coincide. Therefore we do not have to distinguish between $\operatorname{ann}_{R} J_{R}$ and $\operatorname{ann}_{R_{R}} J$, and we will write merely $\operatorname{ann}_{R} J$. Because of this symmetry we will state the theorems of this section for "modules" but only carry out the proofs for right modules.

3.1. LEMMA. Let $\mathscr{N}$ be the set of annihilator ideals of a semi-prime ring $R$. The correspondence $N \rightarrow \operatorname{ann}_{R} N$ is 1-1 and inclusion reversing of $\mathscr{N}$ onto 
$\mathscr{N}$, and is its own inverse. If $A$ and $B$ are arbitrary ideals of $R$ such that $A \subseteq B$, then $\operatorname{ann}_{R} A \supseteq \operatorname{ann}_{R} B$.

We omit the straightforward proof and now state the first main theorem.

3.2. THEOREM. $A$ ring $R$ is an irredundant subdirect sum of prime rings $\left\{R_{\alpha}: \alpha \in A\right\}$ if and only if

(1) $R$ is semi-prime; and

(2) every annihilator ideal of $R$, except $R$ itself, is contained in a maximal annihilator ideal.

When the above conditions are satisfied the set of kernels of the projections $R \rightarrow R_{\alpha}$ coincides with the set of maximal annihilator ideals of $R$. Hence, the $R_{\alpha}$ 's are determined up to isomorphism by $R$.

Proof that the decomposition exists: Assume (1) and (2). Since $R$ is semi-prime $0=\operatorname{ann}_{R} R$ so that $R$ has annihilator ideals different from $R$ (except if $R=0$ in which case the theorem is trivial). Hence, by (2) the set $\left\{P_{\alpha}\right\}$ of maximal annihilator ideals is nonempty. Let $L_{\alpha}=\operatorname{ann}_{R} P_{\alpha}$. Then by Lemma $3.1,\left\{L_{\alpha}\right\}$ is the set of minimal annihilator ideals and $L_{\alpha} \neq L_{\beta}$ for $\alpha \neq \beta$. If $\alpha \neq \beta$, then $\operatorname{ann}_{R}\left(P_{\alpha}+P_{\beta}\right)=0$, for $\operatorname{ann}_{R}\left(P_{\alpha}+P_{\beta}\right) \supset 0$ would imply

$$
P_{\alpha} \subset P_{\alpha}+P_{\beta} \subseteq \operatorname{ann}_{R} \operatorname{ann}_{R}\left(P_{\alpha}+P_{\beta}\right) \subset R
$$

contradicting the maximality of $P_{\alpha}$. Now, $\left(P_{\alpha}+P_{\beta}\right)\left(L_{\alpha} \cap L_{\beta}\right)=0$, and therefore

$$
L_{\alpha} \cap L_{\beta}=0 \quad(\alpha \neq \beta) \text {. }
$$

We now show that $\left\{R / P_{\alpha}\right\}$ is the required set of prime rings. First, $\bigcap_{\alpha} P_{\alpha}=\bigcap_{\alpha} \operatorname{ann}_{R} L_{\alpha}=\operatorname{ann}_{R} \sum_{\alpha} L_{\alpha}$. By Lemma 3.1 and condition (2) of this theorem, every nonzero annihilator ideal of $R$ contains a minimal annihilator ideal. Hence if $\bigcap_{\alpha} P_{\alpha} \neq 0, L_{\beta} \subseteq \operatorname{ann}_{R} \Sigma_{\alpha} L_{\alpha}$ for some $\beta$. But then, by $\left(^{*}\right)$, $0=L_{\beta} \Sigma_{\alpha} L_{\alpha}=L_{\beta}^{2}$, which is impossible since $R$ is semi-prime. Hence $\bigcap_{\alpha} P_{\alpha}=0$. Let $\beta$ be given. Then $\bigcap_{\alpha \neq \beta} P_{\alpha}=\operatorname{ann}_{R} \sum_{\alpha \neq \beta} L_{\alpha} \supseteq L_{\beta} \neq 0$ so that $R$ is an irredundant subdirect sum of $\left\{R / P_{\alpha}\right\}$.

To see that $R / P_{\alpha}$ is a prime ring, suppose that $A B \subseteq P_{\alpha}$ for ideals $A, B$ in $R$. Then $A\left(B L_{\alpha}\right)=0$. If $B L_{\alpha}=0$, then $B \subseteq \operatorname{ann}_{R} L_{\alpha}=P_{\alpha}$. If $B L_{\alpha} \neq 0$, then since $B L_{\alpha} \subseteq L_{\alpha}$, we have, by Lemma 3.1, that $P_{\alpha}=\operatorname{ann}_{R} L_{\alpha} \subseteq \operatorname{ann}_{R}\left(B L_{\alpha}\right) \subset R$. Since $P_{\alpha}$ is a maximal annihilator ideal, $P_{\alpha}=\operatorname{ann}_{R}\left(B L_{\alpha}\right)$ and so $A \subseteq \operatorname{ann}_{R}\left(B L_{\alpha}\right)=P_{\alpha}$. Thus $R / P_{\alpha}$ is a prime ring and we have shown that the decomposition exists.

We will establish the remaining assertions of the theorem in $\$ 3.10$, after deriving some more general results about modules.

3.3. Let a homomorphism $f$ of a ring $R$ onto $S$ be given. We will say that an $R$-module $M_{R}$ is canonically an $S$-module if $M(\operatorname{ker} f)=0$. This condition is satisfied if and only if $M$ becomes an $S$-module when multiplication is defined by $m f(x)=m x(x \in R, m \in M)$.

If $R$ is the direct sum of a finite number of rings $R_{\alpha}$ with identity element $e_{\alpha}$, 
then every unitary $R$-module $M_{R}$ is a direct sum of canonically $R_{\alpha}$-modules: $M=\Sigma_{\alpha} \oplus M e_{\alpha}$ and the decomposition is unique. This statement is not true if direct is replaced by subdirect, as the following example shows:

Let $Z$ be the ring of integers, and $R=\{(x, y): x, y \in Z$, and $x-y \in 2 Z\}$. Then $R$ is a subdirect sum of two copies of $Z$. Let $M=\{(\bar{a}, \bar{b}): \bar{a}, \bar{b} \in Z / 4 Z\}$. Then $M$ becomes an $R$-module if we define $(\tilde{a}, \bar{b})(x, y)=(\bar{a} x, \bar{b} y)$. Let $N=\{(\overline{0}, \overline{0}),(\overline{2}, \overline{2})\}$. Then $N$ is an $R$-submodule of $M$. If $N_{R} \subset Q_{R} \subseteq M_{R}$, a simple computation shows that $(\overline{2}, \overline{0}) \in Q$. This shows that every nonzero submodule of $M / N$ contains $(\overline{2}, \overline{0})+N$ so that $M / N$ is subdirectly irreducible. Since $M / N$ is not itself canonically $(Z, 0)$ or $(0, Z)$-module it follows that $M / N$ cannot be written as a subdirect sum of canonically $(Z, 0)$ - and $(0, Z)$-modules.

On the other hand, $N$ is canonically both a $(Z, 0)$ - and $(0, Z)$-module, and therefore any decomposition of $N \oplus N \oplus N$ as a direct (or subdirect) sum of two modules will be a decomposition into canonically $(Z, 0)$ - and $(0, Z)$-modules.

Now we introduce a condition which will guarantee the existence and uniqueness of canonical decompositions.

3.4. A right $R$-module $M$ is $I$-torsion-free if $N J=0$, for some $N_{R}(\neq 0) \subseteq M$ and some ideal $J$, implies that $K J=0$ for some nonzero ideal $K$. For semi-prime rings this can be restated: if $\operatorname{ann}_{R} J=0$ then $\operatorname{ann}_{M} J=0$ (if $R$ is not semi-prime, the notation $\operatorname{ann}_{R} J$ may be ambiguous). If $R$ is a prime ring, this requires that every nonzero submodule of $M$ be faithful, and if $R$ is a commutative integral domain this definition coincides with the ordinary definition of torsion-free.

3.5. Lemma. Let $R$ be an irredundant subdirect sum of prime rings $\left({ }^{2}\right)$ $\left\{R_{\alpha}\right\}$ and let $J$ be an ideal in $R$. Then $\operatorname{ann}_{R} J=0$ if and only if $J_{\alpha} \neq 0$ for all $\alpha$.

Proof. Suppose $J_{\alpha} \neq 0$ for all $\alpha$ and $J x=0$. Then for all $\alpha, J_{\alpha} x_{\alpha}=0$. Since $R_{\alpha}$ is a prime ring, $x_{\alpha}=0$ for all $\alpha$, i.e., $x=0$. Conversely, if $J_{\alpha}=0$, then $J\left(R \cap R_{\alpha}\right)=0$.

3.6. LemMA. Let $R$ be an irredundant subdirect sum of prime rings $\left\{R_{a}\right\}$ and let $M_{R}$ be canonically an $R_{\beta}$-module for some $\beta$. Then $M$ is I-torsion-free as an $R$-module if and only if it is I-torsion-free as an $R_{\beta}$-module.

Proof. Let $J$ be an ideal in $R$ such that $\operatorname{ann}_{R} J=0$. If $N J=0$ for some $N_{R} \subseteq M$, then $N J_{\beta}=0$. If $M$ is $R_{\beta}$-I-torsion-free, then since $J_{\beta} \neq 0$ (by 3.5) and $R_{\beta}$ is a prime ring, $N=0$ and $M$ is $R$ - $I$-torsion-free. Conversely, suppose $M$ is $R$ - $I$-torsion-free, let $J_{\beta}$ be a nonzero ideal of $R_{\beta}$, and $N$ a submodule of $M$ such that $N J_{\beta}=0$. Then $J_{\beta}\left(R \cap R_{\beta}\right) \neq 0$ since $R_{\beta}$ is a prime ring, and

$$
N\left[J_{\beta}\left(R \cap R_{\beta}\right)+\sum_{\alpha \neq \beta}\left(R \cap R_{\alpha}\right)\right]=0 .
$$

(2) Such a ring is obviously semi-prime, for if $A^{n}=0$ for some ideal $A$, then $A_{\alpha}^{n}=0$ for all $\alpha$, and hence $A=0$. 
Since $M$ is $R$ - $I$-torsion-free, Lemma 3.5 now implies that $N=0$, so that $M$ is $R_{\beta}$-I-torsion-free.

3.7. Because of the above lemma there is no ambiguity in referring to the components $M_{\alpha}$ in the following theorem as canonically I-torsion-free $R_{\alpha^{-}}$ modules.

THEOREM. Let $R$ be an irredundant subdirect sum of prime rings $\left\{R_{\alpha}: \alpha \in A\right\}$ and let $M$ be an I-torsion-free $R$-module. Then $M$ is a subdirect sum of canonically I-torsion-free $R_{\alpha}$-modules $M_{\alpha}(\alpha \in A)$.

Proof. Let( $\left({ }^{3}\right) \quad N_{\alpha}=\operatorname{ann}_{M}\left(R \cap R_{\alpha}\right)$. Then $\bigcap_{\alpha} N_{\alpha}=\bigcap_{\alpha} \operatorname{ann}_{M}\left(R \cap R_{\alpha}\right)$ $=\operatorname{ann}_{M} \Sigma_{\alpha}\left(R \cap R_{\alpha}\right)$. By Lemma 3.5, $\operatorname{ann}_{R} \Sigma_{\alpha}\left(R \cap R_{\alpha}\right)=0$. Since $M$ is $I$-torsionfree, this implies that $\bigcap_{\alpha} N_{\alpha}=0$. Thus $M$ is a subdirect sum of $\left\{M_{\alpha}=M / N_{\alpha}\right\}$. Let $P_{\alpha}=\left\{x \in R: x_{\alpha}=0\right\}$, so that $P_{\alpha}$ is the kernel of the projection $R \rightarrow R_{\alpha}$. Then $\left(M P_{\alpha}\right)\left(R \cap R_{\alpha}\right)=0$ so that $M P_{\alpha} \subseteq \operatorname{ann}_{M}\left(R \cap R_{\alpha}\right)=N_{\alpha}$. Hence $M / N_{\alpha}$ is canonically an $R_{\alpha}$-module.

To see that $M_{\alpha}$ is $I$-torsion-free, suppose $m_{\alpha} J=0$ where $\operatorname{ann}_{R} J=0$. Let $m \in M$ have its $\alpha$ th projection equal to $m_{\alpha}\left(M\right.$ is a subdirect sum of $\left.\left\{M_{\alpha}\right\}\right)$. Then $m\left(R \cap R_{\alpha}\right) J \subseteq m_{\alpha} J=0$. Since $M$ is $I$-torsion-free $m\left(R \cap R_{\alpha}\right)=0$, so that $m \in \operatorname{ann}_{M}\left(R \cap R_{\alpha}\right)=N_{\alpha}$, i.e., $m_{\alpha}=0$. Hence $\operatorname{ann}_{M_{\alpha}} J=0$ and $M_{\alpha}$ is $I$-torsion-free.

3.8. Proposition $\left({ }^{4}\right)$. Let $R$ be an irredundant subdirect sum of prime rings $\left\{R_{\alpha}: \alpha \in A\right\}$, let $M_{R}$ be a subdirect sum of canonically I-torsion-free $R_{\alpha}$-modules, $M_{\alpha}(\alpha \in A)$, and set $A^{\prime}=\left\{\alpha \in A: M_{\alpha}=0\right\}$. Then $M_{R}$ is I-torsion-free and for every ideal $J$ of $R$ and submodule $H$ of $M$ :

(1) $\operatorname{ann}_{M} J=\left\{m \in M: m_{\alpha}=0\right.$ whenever $\left.J_{\alpha} \neq 0\right\}$,

(2) $\operatorname{ann}_{R} H=\left\{x \in R: x_{\alpha}=0\right.$ whenever $\left.H_{\alpha} \neq 0\right\}$,

(3) The correspondence $Z_{M}: B \rightarrow\left\{m \in M: m_{\beta}=0\right.$ for all $\left.\beta \in B\right\}$ is 1-1 and inclusion reversing between the subsets $B$ of $A-A^{\prime}$ and the annihilator submodules of $M$.

Proof. If for some $m \in M, m J=0$, then $m_{\alpha} J_{\alpha}=0$ for each $\alpha$. Hence $J_{\alpha} \neq 0$ implies $m_{\alpha}=0$. Obviously $m J=0$ if $m_{\alpha}=0$ whenever $J_{\alpha} \neq 0$. Thus (1) is established.

If $H x=0$ for some $x$, then $H_{\alpha} x_{\alpha}=0$ for each $\alpha$. Since $R_{\alpha}$ is prime, every nonzero submodule of the $R_{\alpha}$-module $H_{\alpha}$ is faithful. Hence $H_{\alpha} \neq 0$ implies $x_{\alpha}=0$. If $x_{\alpha}=0$ whenever $H_{\alpha}=0$, then $H x=0$ and (2) is established.

(1) shows that $Z_{M}$ is onto. To see that it is $1-1$, let $B$ and $C$ be subsets of $A-A^{\prime}$ such that $B$ contains an element $\beta$ not in $C$. Then $M\left(R \cap R_{\beta}\right) \neq 0$ since $\beta \notin A^{\prime}$. Hence $M\left(R \cap R_{\beta}\right) \ddagger Z_{M}(B)$ but $M\left(R \cap R_{\beta}\right) \subseteq Z_{M}(C)$ so that $Z_{M}(B) \neq Z_{M}(C)$.

(3) In discussions involving subdirect sums, subscripts will be restricted to projections or the kernels of projections. Here $N_{\alpha}$ will be a kernel.

(4) This proposition is due to the referee. 
To see that $M$ is $I$-torsion-free suppose $\operatorname{ann}_{R} J=0$ for some ideal $J$. Then by Lemma $3.5, J_{\alpha} \neq 0$ for all $\alpha$, and by (1) above $\operatorname{ann}_{M} J=0$.

Each $R_{\alpha}$ is a canonically $I$-torsion-free $R_{\alpha}$-module. Hence taking $M=R$ we obtain:

3.9. Corollary. Let $R$ be as above. Then $B \rightarrow Z_{R}(B)$ is a 1-1 inclusion reversing correspondence between the subsets $B$ of $A$ and the annihilator ideals of $R$.

3.10. The completion of the proof of Theorem 3.2 is an immediate consequence of 3.9 , for the kernel of the projection $R \rightarrow R_{\alpha}$ is $Z_{R}(\{\alpha\})$ and the minimal subsets of the index set $A$ are precisely the single-element subsets of $A$. ( $R$ is semi-prime by the footnote to 3.5.) More generally, by applying this reasoning to Proposition 3.8, we obtain the following uniqueness property for modules:

THEOREM. Let $M$ be decomposed as in Theorem 3.7 (M, $M_{\alpha} I$-torsion-free). Then $M$ is an irredundant subdirect sum of those $M_{\alpha}$ 's which are not zero, and the kernels of the projections $M \rightarrow M_{\alpha}\left(M_{\alpha} \neq 0\right)$ are the maximal annihilator submodules of $M$.

Proof. The kernels are characterized as above. To establish irredundance, note that if $M_{\alpha} \neq 0, \alpha \notin A^{\prime}$ ( $A^{\prime}$ is defined in Proposition 3.8) so that $M \cap M_{\alpha}$ $=Z_{M}\left(A-A^{\prime}-\{\alpha\}\right)$ which is not zero since $Z_{M}$ is $1-1$ and $Z_{M}\left(A-A^{\prime}\right)=0$.

3.11. If, in Theorem 3.10, we drop the hypothesis that each $M_{\alpha}$ be $I$-torsionfree, then the decomposition is no longer unique. For we may take $R=\{(x, y)$ : $x, y \in Z, x-y \in 2 Z\}$ ( $Z$ the integers), $M=R, M_{1}=\{(a, \bar{b}): a \in Z, \bar{b} \in Z / 4 Z\}, M_{2}=Z$ (with $m_{2}(x, y)=m_{2} y$ ). Then $M$ is an irredundant subdirect sum of $M_{1}$ and $M_{2}$ : For $(a, b) \in M$, let $f(a, b)=(a, \bar{b} ; b)$. Therefore, for modules we need a stronger condition than irredundance to establish complete uniqueness.

Proposition. Let $M$ be decomposed as in Theorem 3.7. Then each nonzero submodule of each $M_{\alpha}$ has nonzero intersection with $M$. Conversely, if $M$ (which is I-torsion-free) is decomposed into a subdirect sum of canonically $\boldsymbol{R}_{\alpha}$-modules $H_{\alpha}(\alpha \in A)$ having this intersection property, each $H_{\alpha}$ is I-torsion-free (and we may use Theorem 3.10).

Proof. Suppose $0 \neq T_{\beta} \subseteq M_{\beta}$ for some $\beta$, in the decomposition of Theorem 3.7. Then if $M \cap T_{\beta}=0$ we have $0=\left(M \cap M_{\beta}\right) \cap T_{\beta}$. By Proposition 3.8, $M \cap M_{\beta}$ $=\operatorname{ann}_{M} \sum_{\alpha \neq \beta}\left(R \cap R_{\alpha}\right)$. Hence $T_{\beta}\left(\sum_{\alpha \neq \beta}\left(R \cap R_{\alpha}\right)\right) \neq 0$. But this is impossible since $M_{\beta}$ is canonically an $R_{\beta}$-module. Hence $M \cap T_{\beta} \neq 0$.

Conversely, suppose $M$ is decomposed into the $H_{\alpha}$ 's described above. Let $T_{\beta}$ be a submodule of some $H_{\beta}$, and let $J$ be an ideal of $R$ such that $\operatorname{ann}_{R} J=0$. Then if $T_{\beta} J=0,\left(T_{\beta} \cap M\right) J=0$. Since $M$ is $I$-torsion-free, this implies $T_{\beta} \cap M=0$ so that $T_{\beta}=0$. Hence $H_{\beta}$ is $I$-torsion-free. 
3.12. For general subdirect decompositions the components $R_{\alpha}$ of $R$ are not necessarily contained in $R$ (when $R$ is considered as a subring of $\prod_{\alpha} R_{\alpha}$ ). Hence the strongest uniqueness property of such decompositions is uniqueness of the kernels of the projections $R \rightarrow R_{\alpha}$. However, for those subdirect sums of prime rings which contain all of their components we can prove that the projections $R \rightarrow R_{\alpha}$ are unique endomorphisms of $R$.

We will call $R$ an intermediate direct product of a set of its ideals $\left\{L_{\alpha}\right\}$ if there is an isomorphism $h$ of $R$ into the direct product of the rings $\left\{L_{\alpha}\right\}$ such that for $x \in L_{\alpha}, h(x)_{\alpha}=x$ and $h(x)_{\beta}=0(\beta \neq \alpha)$. We will write $r_{\alpha}$ for $h(r)_{\alpha}(r \in R)$.

THEOREM. Let $R$ be an intermediate direct product of a set of nonzero ideals $\left\{L_{\alpha}: \alpha \in A\right\}$ which are prime rings. Then $\left\{L_{\alpha}\right\}$ is the set of minimal annihilator ideals of $R$ and for each $r \in R$ and each $\alpha, r_{\alpha}=\left(r+\operatorname{ann}_{R} L_{\alpha}\right) \cap L_{\alpha}$.

Proof $\left({ }^{5}\right) . \quad L_{\alpha}=Z_{R}(A-\{\alpha\})$ so that by Corollary 3.9, $\left\{L_{\alpha}\right\}$ is the set of minimal annihilator ideals of $R$. Let $r \in R$ and $\alpha$ be given. Since the kernel of the projection $R \rightarrow R_{\alpha}$ is $Z_{R}(\{\alpha\}), r_{\alpha} \in r+Z_{R}(\{\alpha\})$ and $r_{\alpha}$ is then characterized by the further requirement $\left(r_{\alpha}\right)_{\beta}=0(\beta \neq \alpha)$, i.e., $r_{\alpha}=r+Z_{R}(\{\alpha\}) \cap L_{\alpha}$. By Proposition 3.8 (with $M=R$ ), $\operatorname{ann}_{R} L_{\alpha}=Z_{R}(\{\alpha\})$, and this completes the proof.

3.13. The results of this section enable us to characterize all finite subdirect sums of prime rings.

THEOREM. The following conditions are equivalent:

(1) $R$ is a subdirect sum of a finite number of prime rings.

(2) $R$ is an irredundant subdirect sum of a finite number of prime rings.

(3) $R$ is a semi-prime with the maximum condition for annihilator ideals.

Proof. (1) and (2) are equivalent for arbitrary (not necessarily prime) rings, for if $R$ is a subdirect sum of a finite number of rings $R_{i}(i=1, \ldots, n)$ and if the sum is not irredundant, then for some $j$, the map $r \rightarrow\left\{r_{i}: i \neq j\right\}$ is $1-1$. $R$ is now expressed as a subdirect sum of $n-1$ rings, and if this sum is not irredundant we can repeat the process until we obtain one which is.

If (2) holds then $R$ is semi-prime by Theorem 3.2. By Corollary 3.9, $R$ has only a finite number of annihilator ideals. Therefore (3) holds.

On the other hand, if (3) holds, then by Theorem 3.2, $R$ is an irredundant subdirect sum of prime rings $\left\{R_{\alpha}: \alpha \in A\right\}$, and by Corollary 3.9, the index set $A$ satisfies the minimum condition for subsets. Since this is equivalent to the descending chain condition for subsets, $A$ is a finite set.

3.14. EXAMPLE. We close this section by observing that not every semi-prime ring satisfies the condition every annihilator ideal (different from the ring) is contained in a maximal annihilator ideal: The set of annihilator ideals of

(5) This proof is due to the referee. 
the ring $R$ of continuous, real-valued functions on $[0,1]$ is in $1-1$ inclusionpreserving correspondence with the interiors of closed subsets of $[0,1]$ (see Lambek [4], last paragraph in $\S 6$, and Example 7.5). Hence $R$ has no maximal annihilator ideals.

4. Injective envelopes and maximal quotient rings. Let $M$ be a right $R$-module. We will denote by $M^{*}$ an injective envelope of $M$, that is, a minimal injective module containing $M$. R. E. Johnson [3, Theorem 7.1] has shown the existence and uniqueness, up to isomorphism over $\left(^{6}\right) M$, of $M^{*}$ for rings without identity. An injective module $T^{*}$ containing $M$ is an injective envelope of $M$ if for every nonzero submodule $H$ of $T^{*}, H \cap M \neq 0$.

4.1. Let $R$ be an irredundant subdirect sum of prime rings $\left\{R_{\alpha}: \alpha \in A\right\}$, and let $M$ be an $I$-torsion-free right $R$-module. Then $M$ is an irredundant subdirect sum of canonically $I$-torsion-free $R_{\alpha}$-modules $M_{\alpha}(\alpha \in A)$ by Theorem 3.7.

Proposition. Let $M_{\alpha}^{*}$ be an injective envelope of the $R_{\alpha}$-module $M_{\alpha}$. Then $M^{*} \cong \prod_{\alpha} M_{\alpha}^{*}$.

Proof. We carry out the proof in three parts.

(1) $M^{*}$ is $I$-torsion-free: If $H$ is a submodule of $M^{*}$ and $J$ an ideal of $R$ such that $\operatorname{ann}_{R} J=0$, then if $H J=0,(H \cap M) J=0$. Since $M$ is $I$-torsion-free $H \cap M=0$ so that $H=0$.

(2) $M_{\alpha}^{*}$ is an injective envelope of the $R$-module $M_{\alpha}$ : Let $T^{*}$ be an injective envelope of the $R$-module $M_{\alpha}$. Let $P_{\alpha}=\left\{x \in R: x_{\alpha}=0\right\}$. If $T^{*} P_{\alpha} \neq 0$, then $\left(T^{*} P_{\alpha}\right) \cap M_{\alpha} \neq 0$. But then $\left(T^{*} P_{\alpha} \cap M_{\alpha}\right)\left(R \cap R_{\alpha}\right) \subseteq T^{*} P_{\alpha}\left(R \cap R_{\alpha}\right)=0$ contradicting the fact that $M_{\alpha}$ is an $I$-torsion-free $R_{\alpha}$-module. Hence $T^{*} P_{\alpha}=0$ and $T^{*}$ is canonically an $R_{\alpha}\left(\cong R / P_{\alpha}\right)$-module. It now follows easily that $T^{*}$ is an injective envelope of the $R_{\alpha}$-module $M_{\alpha}$. $\left(R\right.$-injective implies $R_{\alpha}$-injective. Then use the intersection property of injective envelopes.) The desired conclusion follows from the uniqueness of injective envelopes.

(3) Let $T^{*}=\prod_{\alpha} M_{\alpha}^{*}$. There is a natural embedding of $M$ in $T^{*}$ since for each $\alpha, M_{\alpha} \subseteq M_{\alpha}^{*}$. We show that $T^{*} \cong M^{*}$ (isomorphism over $M$ ): Since a product of injective modules is injective [1, Chapter 1] (the proof remains unchanged for rings without identity), (2) implies that $T^{*}$ is injective. Each $M_{\alpha}^{*}$ is canonically $I$-torsion-free by (1) and Lemma 3.6. Hence $T^{*}$ is $I$-torsion-free (Proposition 3.8). Let $H$ be a nonzero submodule of $T^{*}$. Then $\operatorname{ann}_{R} \Sigma_{\alpha}\left(R \cap R_{\alpha}\right)=0$ (Lemma 3.5) so that $H \sum_{\alpha}\left(R \cap R_{\alpha}\right) \neq 0$ since $T^{*}$ is $I$-torsion-free. Hence for some $\alpha$, $0 \neq H\left(R \cap R_{\alpha}\right) \subseteq M_{\alpha}^{*}$ so that $H\left(R \cap R_{\alpha}\right) \cap M_{\alpha} \neq 0$. Then by Proposition 3.11, $H\left(R \cap R_{\alpha}\right) \cap M_{\alpha} \cap M \neq 0$, so that $H \cap M \neq 0$. Hence $T^{*}$ is an injective envelope of $M$.

4.2. Let $\left\{R_{\alpha}\right\}$ be a set of rings with zero left annihilator, and let $S_{\alpha}$ be a maximal

(6) Two modules $H$ and $K$ containing $M$ are isomorphic over $M$ if the identity map on $M$ can be extended to an isomorphism of $H$ onto $K$. 
ring of right quotients of $R_{\alpha}$. Utumi $[5,2.1]$ has shown that $\prod_{\alpha} S_{\alpha}$ is a maximal ring of right quotients of $\Sigma \oplus R_{\alpha}$. Lambek [4, Proposition 7.2] has proved the two-sided analogue of this by assuming, in addition, that each $R_{\alpha}$ has zero right annihilator. We now show that if the $R_{\alpha}$ 's are prime rings, both of these results can be considerably strengthened.

Proposition. Let $S_{\alpha}$ be a maximal ring of right (resp. right and left) quotients of the prime ring $R_{\alpha}(\alpha \in A)$ and let $R$ be an irredundant subdirect sum of $\left\{R_{\alpha}: \alpha \in A\right\}$. Then $\prod_{\alpha} S_{\alpha}$ is a maximal ring of right (resp. right and left) quotients of $R$.

Proof. For the one-sided version we adopt the terminology and notation of Utumi [5]. Since $\operatorname{ann}_{R} \sum_{\alpha}\left(R \cap R_{\alpha}\right)=0$ and $\operatorname{ann}_{R_{\alpha}}\left(R \cap R_{\alpha}\right)=0$ (Lemma 3.5), we have

(1) $R \geqq \Sigma_{\alpha}\left(R \cap R_{\alpha}\right)$,

(2) $R_{\alpha} \geqq R \cap R_{\alpha}$ for each $\alpha$.

By (2), $\bar{R}_{\alpha}$ is a maximal ring of right quotients of $R \cap R_{\alpha}[5,1.14]$, so that $\prod_{\alpha} \bar{R}_{\alpha}$ is a maximal ring of right quotients of $\Sigma_{\alpha}\left(R \cap R_{\alpha}\right)=\Sigma_{\alpha} \oplus\left(R \cap R_{\alpha}\right)$. Then by (1), $\bar{R} \cong \prod_{\alpha} \bar{R}_{\alpha}$.

For the two-sided version, we note that parts (1) and (3) of Lambek's proof [4, Proposition 7.2] apply to our case without change. Hence it is sufficient to show that if $T_{\alpha}$ is a ring of right quotients of $R_{\alpha}$, and $T=\prod_{\alpha} T_{\alpha}$, then $R \leqq T\left(T_{R}\right)$. Let $t^{\prime}(\neq 0)$ and $t \in T$ be given. Then for some $\alpha, t_{\alpha}^{\prime} \neq 0$ and since $T_{\alpha}$ is a ring of right quotients of $R_{\alpha}$ there is an $x_{\alpha} \in R_{\alpha}^{*}$ such that $t^{\prime} x_{\alpha}=t_{\alpha}^{\prime} x_{\alpha} \neq 0$ and $t x_{\alpha}$ $=t_{\alpha} x_{\alpha} \in R_{\alpha}$. Since $T_{\alpha}$ is a rational extension of $R_{\alpha}$, it is an essential extension, and we show as in part (1) of Proposition 4.1 that $T_{\alpha}$ is $I$-torsion-free as an $R_{\alpha^{-}}$ module. Hence $t_{\alpha}^{\prime} x_{\alpha}\left(R \cap R_{\alpha}\right) \neq 0$ and for some $y \in R \cap R_{\alpha}$ we have $x_{\alpha} y \in R$, $t^{\prime}\left(x_{\alpha} y\right) \neq 0$, and $t\left(x_{\alpha} y\right) \in R$, giving the desired result.

\section{Square roots and cancellation.}

5.1 Proposition. If $A$ and $B$ are prime rings such that an irredundant subdirect sum of some (possibly infinite) set of copies of $A$ is isomorphic to an irredundant subdirect sum of some set of copies of $B$, then $A \cong B$.

Proof. Let $R$ be the irredundant subdirect sum of copies of $A$ which is also an irredundant subdirect sum of copies of $B$, and then use the uniqueness part of Theorem 3.2.

The "cancellation" theorem, if $A, B$, and $C$ are prime rings such that an irredundant subdirect sum of $\{A, C\}$ is isomorphic to an irredundant subdirect sum of $\{B, C\}$, then $A \cong B$, also follows immediately from Theorem 3.2. A less trivial cancellation result is the following:

5.2. Proposition. Let $C$ be a semi-prime ring satisfying the maximum 
condition for two-sided ideals. If $A$ and $B$ are prime rings such that an irredundant subdirect sum of $\{A, C\}$ is isomorphic to an irredundant subdirect sum of $\{B, C\}$, then $A \cong B$.

The proof will require two lemmas.

5.3. LEMMA. Let $R$ be an irredundant subdirect sum of the rings $A$ and $C$ where $C$ is an irredundant subdirect sum of prime rings $C_{1}, \ldots, C_{n}$. Then the $C_{i}$ 's can be renumbered so that for some $s \leqq n$ the map $h\left(a, c_{1}, c_{2}, \ldots, c_{n}\right)$ $=\left(a, c_{1}, \ldots, c_{s}\right)$ expresses $R$ as an irredundant subdirect sum of $\left\{A, C_{1}, \ldots, C_{s}\right\}$. If $s<n$, then for each $m$ such that $s<m \leqq n, h^{-1}$ induces a homomorphism $f_{m}:$ A onto $C_{m}$ and $\operatorname{ker} f_{m} \neq 0$.

Proof. Let $C_{0}=A$. If $R$ is not already expressed as an irredundant subdirect sum of $\left\{C_{0}, \ldots, C_{n}\right\}$, then for some $j$ the map $h_{(j)}: r \rightarrow\left\{r_{i}: i \neq j\right\}$ of $R$ into $\prod_{i \neq j} C_{i}$ is 1-1. By irredundance of the sum $\{A, C\}$ there is an element $r=\left(c_{0}, c\right)$ $=\left(c_{0}, c_{1}, c_{2}, \ldots, c_{n}\right) \in R$ such that $c_{0} \neq 0$ but $c_{i}=0$ for $i>0$. Then $h_{(0)}\left(c_{0}\right)=0$ so that $h_{(0)}$ is not $1-1$ and hence $j \neq 0$. Now we interchange $C_{j}$ and $C_{n}$ so that $h_{(n)}$ expresses $R$ as a subdirect sum of $\left\{C_{0}, \ldots, C_{n-1}\right\}$. If this sum is not irredundant we can repeat the process until we obtain one which is.

It is sufficient to prove the remainder of the lemma for the case $m=n$. Now, $h^{-1}$ followed by the projection of $R$ onto its $n$th component gives a homomorphism $f$ of $R^{\prime}=h(R)$ onto $C_{n}$. Let $P_{i}=\left\{x \in R^{\prime}: x_{i}=0\right\}$. Then $P_{0} P_{1} \ldots P_{s}=0$ so that $f\left(P_{0}\right) \ldots f\left(P_{s}\right)=0$. Since $C_{n}$ is a prime ring, this implies that $f\left(P_{j}\right)=0$ for some $j$. Since $P_{j}$ is the kernel of the projection of $R^{\prime}$ onto its $j$ th component, $f$ induces a homomorphism $f_{n}$ of $C_{j}$ onto $C_{n}$. Since $C$ is an irredundant subdirect sum of $\left\{C_{1}, \ldots, C_{n}\right\}$ there is an element $c=\left(0_{1}, 0_{2}, \ldots, 0_{n-1}, c_{n}\right) \in C$ with $c_{n} \neq 0$. Since $R$ is a subdirect sum of $\left\{C_{0}, C\right\}$, there is a $c_{0} \in C_{0}$ such that $\left(c_{0}, c\right)=\left(c_{0}, 0_{1}, \ldots, 0_{n-1}, c_{n}\right) \in R$. Then $j \neq 0$ would imply $f_{n}\left(0_{j}\right)=c_{n} \neq 0$, which is impossible. Hence $j=0$ and $f_{n}$ maps $C_{0}=A$ onto $C_{n}$. Since $R$ is an irredundant subdirect sum of $\{A, C\}$ there is an element $\left(r_{0}, 0_{1}, \ldots, 0_{n}\right) \in R$ with $r_{0} \neq 0$. Thus $f_{n}\left(r_{0}\right)=0$ and $\operatorname{ker} f_{n} \neq 0$.

5.4. LEMMA. Let $R$ be a ring satisfying the ascending chain condition for two-sided ideals. Then every (ring) homomorphism of $R$ onto itself is 1-1.

Proof. Let $f$ be a homomorphism of $R$ onto $R$. If $f$ were not 1-1 we would have the strictly ascending chain of ideals $0 \subset f^{-1}(0) \subset f^{-2}(0) \subset \ldots$ contradicting the ascending chain condition.

Proof of Proposition 5.2. Let $R$ be the irredundant subdirect sum of $A$ and $C$ which is also an irredundant subdirect sum of $B$ and $C$. By Theorem 3.13, $C$ is an irredundant subdirect sum of a finite number of prime rings $C_{i}(i=1,2, \ldots, n)$. To simplify the following discussion we suppose all of the rings $A, B, C_{1}, \ldots, C_{n}$ to be distinct from each other (they still might be isomorphic to each other). 
By Lemma 5.3 we can renumber the $C_{i}$ 's so that $R$ is an irredundant subdirect sum of $S_{1}=\{A\} \cup\left\{C_{1}, \ldots, C_{s}\right\}$ for some $s \leqq n$. By the same lemma, there is a subset $J_{2}$ of $\{1,2, \ldots, n\}$ such that $R$ is an irredundant subdirect sum of $S_{2}=\{B\} \cup\left\{C_{i}: i \in J_{2}\right\}$.

For each set $S$ of rings let $a(S)$ be the number of rings in $S$ which are isomorphic to $A$. Then since the prime components of $R$ are unique up to isomorphism (Theorem 3.2), $a\left(S_{1}\right)=a\left(S_{2}\right)$. Suppose, now, that $A$ is not isomorphic to $B$. Then for some $m>s, A \cong C_{m}$ : for otherwise $a\left(S_{2}\right)=a\left(\left\{C_{i}: i \in J_{2}, i \leqq s\right\}\right)$ $\leqq a\left(\left\{C_{i}: i \leqq s\right\}\right)=a\left(S_{1}\right)-1$, a contradiction. Let $g$ be an isomorphism of $C_{m}$ onto $A$ and let $f_{m}$ be the homomorphism of $A$ onto $C_{m}$ given by Lemma 5.3. Then $f_{m} g$ is a homomorphism of $C_{m}$ onto itself with nonzero kernel. But $C_{m}$, being a homomorphic image of $C$, satisfies the maximum condition for twosided ideals, so that by Lemma 5.4 we cannot have $\operatorname{ker} f_{m} g \neq 0$. Hence $A \cong B$.

5.5. It might seem, in Proposition 5.2, that the maximum condition could be replaced by the weaker requirement that $C$ have a finite number of prime components. However, this is not the case, as the following example shows:

Let $A$ be a polynomial ring in an infinite number of indeterminates over the integers, and let $B$ be the same polynomial ring over the rationals. Then there is a homomorphism $f$ (resp. g) of $A$ (resp. $B$ ) onto itself with a nonzero kernel. Let $C=A \oplus B$. Then $R=\{(x ; y, z): x \in A,(y, z) \in C, f(x)=y\}$ is a subdirect sum of $A$ and $C$. The sum is irredundant since if $k$ is a nonzero element of ker $f$, $(k ; 0,0) \in R$ and $(0 ; 0, z) \in R$ for each nonzero $z \in B$. However, $R \cong C$ since the middle component can be omitted. Similarly we can construct an irredundant subdirect sum of $B$ and $C$ which is isomorphic to $C$. Since $A$ is not isomorphic to $B$, we have the desired example.

6. Classical quotient rings. By a (classical) right quotient ring of a ring $R$, we mean a ring $S$ with identity such that $S \supseteq R$, every regular element (i.e., non-zerodivisor) $d$ of $R$ has a two-sided inverse $d^{-1}$ in $S$, and every element of $S$ has the form $r d^{-1}$ for $r, d \in R$. It is easily seen that if $S$ exists it is unique up to isomorphism over $R$.

Suppose that $S$ exists and is semi-simple with minimum condition. Then by a result of Goldie [2] $R$ is semi-prime and satisfies the ascending chain condition for annihilator ideals. Then by Theorem $3.13, R$ is an irredundant subdirect sum of a finite number of prime rings $\left\{R_{i}\right\}$, and it is natural to inquire whether each $R_{i}$ has a right quotient ring.

If $R$ has a right quotient ring which is semi-simple (resp. simple) with minimum condition, we will say that $R$ is rqss (resp. rqs).

6.1. THEOREM. $R$ is rqss if and only if it is an irredundant subdirect sum of a finite number of rqs rings $\left\{R_{i}\right\}$. The kernels of the projections $R \rightarrow R_{i}$ are the maximal annihilator ideals of $R$. 
Before beginning the proof we note that if $R$ is rqs, then $R$ is a prime ring (by [2, Theorem 5.1 and Lemma 5.13] the only annihilator ideals of $R$ are $R$ and 0$)$.

6.2. Lemma. Every nonzero (two-sided) ideal in an rqs ring contains a regular element.

Proof. Let $J$ be the given ideal of $R$. If $K$ is an arbitrary nonzero right ideal, then $J \cap K \supseteq K J \neq 0$ since a nonzero ideal of a prime ring has zero annihilator. But a theorem of Goldie [2, Theorem 3.9] states that a right ideal $J$ of an rqs ring $R$ has regular elements if and only if $J \cap K \neq 0$ for every nonzero right ideal $K$ of $R$.

6.3. Lemma. Let $R$ be an irredundant subdirect sum of rqs rings $\left\{R_{i}\right\}$. If an element $d$ of $R$ is regular then $d_{i}$ is regular (in $R_{i}$ ) for each $i$.

Proof. Let $d$ be regular in $R$ and suppose that for some $x \in R, x_{i} d_{i}=0$. Then $0=\left(R \cap R_{i}\right) x_{i} d_{i}=\left(R \cap R_{i}\right) x d$. Hence, since $d$ is regular, $0=\left(R \cap R_{i}\right) x$ $=\left(R \cap R_{i}\right) x_{i}$. By irredundance, $R \cap R_{i} \neq 0$. Hence, since $R_{i}$ is prime, $x_{i}=0$. Similarly $d_{i}$ is not a left zero-divisor.

6.4. LemMa. Let $S$ be a simple ring with minimum condition. If $d \in S$ is not a left zero-divisor, then $d$ is invertible.

Proof. We may consider $S$ to be the complete ring of linear transformations of a finite dimensional vector space $V$ (right operators). If $V d \neq V$, then for some $x \neq 0, V d x=0$. But $d x=0$ implies $x=0$, a contradiction. Hence $V d=V$, and since $V$ is finite dimensional, $d$ is also $1-1$. Hence $d^{-1} \in S$.

Proof of Theorem 6.1 $\left({ }^{7}\right)$. Let $R$ be rqss and let $S$ be the right quotient ring of $R$. If $R=0$ then $R$ is an irredundant subdirect sum of the empty set of rqs rings. From now on suppose $R \neq 0$. Let $S=\sum_{i=1}^{n} \oplus S_{i}$ where each $S_{i}$ is a simple ring, and let $e_{i}$ be the identity of $S_{i}$. For $s \in S$, let $s_{i}=s e_{i}$, and let $R_{i}=R e_{i}$. Then $R$ is a subdirect sum of the set of rings $\left\{R_{i}\right\}$. The sum is irredundant since $e_{i}=x d^{-1}(x, d \in R)$ so that $0 \neq d e_{i}=x \in R \cap R_{i}$. To complete the proof of the existence of the decomposition we show that $S_{i}$ is a right quotient ring of $R_{i}$.

Let $d_{i} \in R_{i}$ be regular (in $R_{i}$ ) and suppose $d_{i} s_{i}=0$ for $s_{i} \in S_{i}$. Then $s_{i}=x c^{-1}$ $(x, c \in R)$ so that $0=d_{i} x=d_{i} x_{i}$. Since $d_{i}$ is regular in $R_{i}, x_{i}=0$ so that $s_{t}=0$. Hence $d_{i}$ is not a left zero-divisor in $S_{i}$, and by Lemma 6.4, $d_{i}$ is invertible in $S_{i}$. If $s_{i} \in S_{i}$ is given, then $s_{i}=x d^{-1}=x_{i} d_{i}^{-1}\left(x, d \in R, d_{i}^{-1}\right.$ being the inverse of $d_{i}$ in $S_{i}$ ) and $S_{i}$ is a right quotient ring of $R_{i}$.

Conversely, suppose that $R$ is an irredundant subdirect sum of rqs rings $R_{i}$ $(i=1, \ldots, n)$. Let $S_{\iota}$ be a right quotient ring of $R_{i}$ and set $S=\Sigma \oplus S_{i}$. Let $d \in R$ be regular. Then by Lemma 6.3 each $d_{i}$ is regular in $R_{i}$ and hence invertible in

(7) The author is indebted to the referee for several improvements in this proof. 
$S_{i}$, showing that $d$ is invertible in $S$. Let $s \in S$. Then for each $i, s_{i}=x_{i} d_{i}^{-1}$ $\left(x_{i}, d_{i} \in R_{i}\right)$. By Lemma 6.2, each $R \cap R_{i}$ contains a regular element $c_{i}$ of $R_{i}$. Then $s=\left(x_{1} c_{1}+\ldots+x_{n} c_{n}\right)\left(d_{1} c_{1}+\ldots+d_{n} c_{n}\right)^{-1}$ where $\sum x_{i} c_{i}$ and $\sum d_{i} c_{i}$ both belong to $R$. Hence the semi-simple ring $S$ is a right quotient ring of $R$.

Uniqueness follows from Theorem 3.2. We have actually obtained a characterization of the $R_{i}$ 's in terms of $S$ :

6.5. CoROllary. Let $R$ be rqss with right quotient ring $S$, and write $S=\Sigma \oplus S_{i}$ where each $S_{i}$ is a simple ring with identity element $e_{i}$. Then the rqs components of $R$ are given by $R_{i}=R e_{i}$. Hence $S$ depends only on the components $R_{i}$ and not on the irredundant subdirect sum which combines them.

6.6. REMARKS. If, in the above corollary, $S$ is a two-sided (i.e., left and right) quotient ring of $R$, then each $S_{i}$ is a two-sided quotient ring of $R_{i}$, for $R_{i}=R e_{i}$ $=e_{i} R$ and the proof of Theorem 6.1 can be repeated interchanging left and right.

We may note the similarity between the last assertion of Corollary 6.5 and the results of $\$ 4$. The results are formally related in the following way: Let $R$ be rqss with right quotient ring $S$. Then every homomorphism $f$ of a right ideal $J$ of $R$ into $S$ is given by left multiplication $x \rightarrow a x$ by some $a \in S$ (extend $f$ to an $S$-homomorphism $f^{*}$ of $J S$ into $S$ by $f^{*}(x s)=f(x) s$ and then to $S$ by the fact that $J S$ is a direct summand of $S$ ). Hence, if $R$ is rqss, its right quotient ring is a maximal ring of right quotients of $R$ (in the sense of $\$ 4$ ). It also follows from this that $S_{R}$ is injective so that both of the results of $\S 4$ apply to $R$ and $S$. It would therefore be interesting to know whether or not Theorem 6.1 also holds for maximal quotient rings. It is not clear, however, whether semi-simplicity of the maximal ring of right quotients of $R$ implies that $R$ is semi-prime. Another hypothesis may be needed.

\section{REFERENCES}

1. H. Cartan and S. Eilenberg, Homological algebra, Princeton Univ. Press, Princeton, N.J., 1956.

2. A. W. Goldie, Semi-prime rings with maximum condition, Proc. London Math. Soc. (3) 10 (1960), 201-220.

3. R. E. Johnson, Structure theory of faithful rings. II, Trans. Amer. Math. Soc. 84 (1957), 523-544.

4. J. Lambek, On the structure of semi-prime rings and their rings of quotients, Canad. J. Math. 13 (1961), 392-417.

5. Y. Utumi, On quotient rings, Osaka Math. J. 8 (1956), 1-18.

UNIVERSITY OF WISCONSIN, MADISON, Wisconsin 